\title{
The Secrets of Life in Boiling Acids: Atomic Structure of Sulfolobus Turreted Icosahedral Virus
}

David Veesler ${ }^{1}$, Thiam-Seng $\mathrm{Ng}_{5,6}^{2,3}$, Anoop K. Sendamarai ${ }^{4,5}$, Brian J. Eilers ${ }^{4,5}$, C. Martin Lawrence ${ }^{4,5}$, See-Mei Lok ${ }^{2,3}$, Mark J. Young ${ }^{5,6}$, John E. Johnson ${ }^{1}$ and Chi-yu Fu ${ }^{1}$.

1. Department of Integrative Structural and Computational Biology, The Scripps Research Institute, La Jolla, California, USA

2. Program in Emerging Infectious Diseases, Duke-NUS Graduate Medical School, KTP Building, 8 college Road, Singapore

3. Center for Bioimaging Sciences, National University of Singapore, Singapore

4. Department of Chemistry and Biochemistry, Montana State University, Bozeman, Montana, USA.

5. Thermal Biology Institute, Montana State University, Bozeman, Montana, USA

6. Department of Plant Sciences and Plant Pathology, Montana State University, Bozeman, Montana, USA

Although many Archaea live in so-called extreme ecosystems, viruses infecting extremophile organisms are ubiquitous, suggesting that all forms of life are targeted by viral attacks. Archaeal viruses are characterized by their exceptional morphologies (droplets, fusiforms and bottle-shaped) and unique genetic features (proteins encoded in their genomes are generally unrelated to those of other known viruses) that are believed to reflect their adaptation to hostile ecological niches (1). However, recent structural studies revealed that apparently unrelated viruses share common architectural principles and topologically identical coat subunit folds (2-5). These results led to the definition of the PRD1Adenovirus lineage that allowed for the first time to propose a common evolutionary origin for a protein component belonging to viruses infecting organisms of the three Domains of Life.

Sulfolobus turreted icosahedral virus (STIV) infects the hyperthermo-acidophilic archeon Sulfolobus solfataricus, which grows optimally at $\mathrm{pH} 3$ and $80^{\circ} \mathrm{C}(6)$. STIV is a $75 \mathrm{MDa}$ dsDNA virus assembled from 9 structural proteins and belonging to the PRD1-Adenovirus lineage. Tight control of virus assembly is achieved by modulating the expression pattern of viral and host genes to produce quasicrystalline lattices made of mature virions in the Sulfolobus cytoplasm (7). At the end of the infection cycle, STIV particles are released via an unprecedented mechanism involving the formation of pyramidlike structures perforating the S-layer at the cell surface (7). Despite extensive efforts carried out to decipher the STIV life cycle, little is known about the detailed architecture of this large membranecontaining virion or on any related archaeal virus (8). Moreover, the mechanisms governing its assembly are poorly understood due to the limited resolution of the structural data available.

We present the high-resolution structure of the mature STIV virion obtained using a combination of single particle electron cryomicroscopy and X-ray crystallography of isolated capsid gene products (Figure $1 \mathrm{~A}-\mathrm{B}$ ). The reconstruction allowed identification of many structural proteins as well as tracing of their polypeptide chains due to well-resolved features in the density (the resolution of the reconstruction is estimated to $3.9 \AA$ in the capsid shell region, Figure 1 C). STIV virions are $960 \AA$-wide particles comprising a $730 \AA$ icosahedral capsid decorated with a turret structure at each 5-fold vertex (Figure $1 \mathrm{~A}-\mathrm{B}$ ). The virus architecture is based on a pseudo $\mathrm{T}=31 \mathrm{~d}$ capsid symmetry with each icosahedral asymmetric unit encompassing 15 copies of the coat subunit (B345), one copy of the A223 penton base protein, one copy of the C381 turret protein and one copy of the A55 membrane protein. A 
lipidic membrane is present below the capsid shell and encloses the dsDNA genome, which fills the volume defined by it. Analysis of the architecture of the STIV proteins and their interactions as well as integration of data from the literature conducted us to propose that the vertex complexes coordinate the co-assembly of the lipid membrane and the coat protein shell in the host cytoplasm to form a provirion into which the genome is subsequently packaged. The observed structural conservation of a large number of proteins playing key roles in the architecture and function of viruses belonging to the PRD1Adenovirus lineage constitutes a strong argument in favor of a common ancestor for these virions infecting organisms of the three Domains of Life. Finally, these results reveal that the STIV coat subunit, penton base and turret proteins are made of a succession of fused jelly-roll motifs exhibiting similar folds with a common topology. Based on these observations, we speculate that a unique ancestral protein module gave rise to many of the STIV structural proteins by duplication and sequence diversification events that occurred during a lengthy evolutionary process.

[1] D Prangishvili et al, Nature reviews Microbiology 4 (2006) p. 837-848.

[2] R Khayat et al, Proceedings of the National Academy of Science 102 (2005), p. 18944-18949.

[3] NGA Abrescia et al, Nature 432 (2004), p. 68-74.

[4] NGA Abrescia et al, Molecular Cell 31 (2008), p. 749-761.

[5] JJ Rux et al, Journal of Virology 77 (2003), p. 9553-9566.

[6] G Rice et al, Proceedings of the National Academy of Science 101 (2004), p. 7716-7720.

[7] C-Y Fu et al, Structure 18 (2010), p. 1579-1586.

[8] R Khayat et al, Journal of Virology 84 (2010), p. 9575-9583.
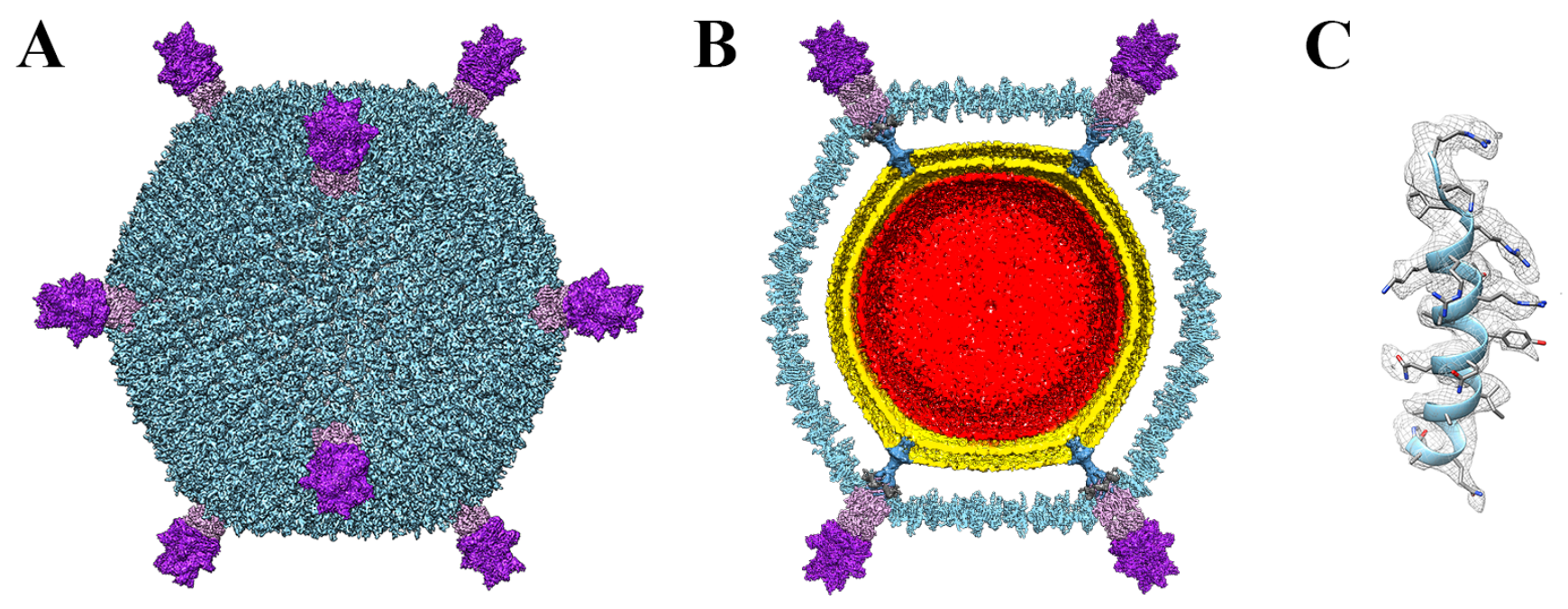

Figure 1. Near-atomic resolution electron cryo-microscopy reconstruction of STIV. (A) The virus reconstruction is shown with the different protein components individually colored (B345: light blue, A223: light pink, C381: purple). (B) Cross-section of the reconstruction revealing the presence of the viral membrane (gold) and the internal genome (red). The cement protein (dark grey) has been removed from the top-right vertex complex to allow visualizing the A223/A55 $\beta$-pore. (C) The coat subunit (B345) C-terminal $\alpha$-helix (residues 325-345) was modeled de novo in the cryoEM density and illustrates the quality of the reconstruction. 